\title{
Bilateral Extracranial Carotid Artery Aneurysms: A Rare Complication of Marfan Syndrome
}

\author{
Lee Mendenhall, MD1', Karl G. Shaver, MD', Lynne J. Goebel, MD, FACP1
}

\section{ABSTRACT}

Marfan syndrome (MFS) is an autosomal dominant connective tissue disorder caused by a mutation in the FBN1 gene, which encodes for the extracellular matrix protein fibrillin-1. The MFS clinical manifestations that lead to morbidity and mortality are primarily those involving aortic disease, such as aneurysmal dilation, aortic regurgitation, and aortic dissection. Despite the fact that $60-80 \%$ of adults with MFS have some degree of aortic disease, carotid artery aneurysms are rare and usually represent extensions of aortic dissections. 'We report a 70-year-old female with a history of ectopia lentis and a family history of MFS who presented to the emergency department with epistaxis and anemia. An arteriogram revealed an incidental finding of bilateral carotid artery aneurysms, a rare phenomenon even in the presence of connective tissue disease. Review of the literature revealed very little data on the clinical presentation, treatment, and outcome of MFS patients with extracranial carotid artery aneurysms (ECAAs). We discuss presentation, etiology, and management options reported for these rare occurrences.
Author affiliations are listed at the end of this article.

Correspondence to: Lynne J. Goebel, MD, FACP Marshall University Joan C. Edwards School of Medicine goebel@marshall.edu

\section{KEYWORDS}

Marfan syndrome, FBN1, fibrillin-1, computed tomography angiography, extracranial carotid artery aneurysms

\section{INTRODUCTION}

Extracranial carotid artery aneurysms (ECAAs) are an uncommon pathology, and bilateral ECAAs represent 6-12\% of carotid artery aneurysms. ${ }^{2-5}$ There can be significant diversity in the presentation of ECAAs; however, due to the frequent use of imaging in modern medicine, their discovery as an incidental finding in asymptomatic patients is increasing. The etiology of ECAAs is quite diverse and includes connective tissue diseases, such as Marfan syndrome (MFS), Ehlers-Danlos syndrome, osteogenesis imperfecta, and pseudoxanthoma elasticum. ${ }^{6}$ MFS is caused by a mutation in the FBN1 gene that encodes for the extracellular matrix protein fibrillin-1 and has an autosomal dominant inheritance pattern. Despite the link between connective tissue disease and ECAAs, the incidence of ECAAs in MFS patients is actually quite low. ${ }^{7,8}$ Furthermore, there is limited research on best-practice management for these patients. We present a case of asymptomatic bilateral ECAAs in a patient who had a history suggestive of MFS and was managed with conservative therapy.

\section{CASE REPORT}

A 70-year-old female presented to the emergency department with uncontrolled epistaxis. Her past medical history included hypertension, hyperlipidemia, hypothyroidism, type 2 diabetes mellitus, and ectopia lentis. Upon arrival, a complete blood count revealed a hemoglobin $(\mathrm{Hgb})$ of $10.8 \mathrm{~g} / \mathrm{dL}$. The epistaxis initially responded to sterile tissue packing; however, she subsequently developed two recurrent episodes of epistaxis accompanied by a decrease in her $\mathrm{Hgb}$ to $9.2 \mathrm{~g} / \mathrm{dL}$. Cauterization of the right internal maxillary artery failed to control the bleeding; therefore, an arterial embolization was planned. Computed tomography angiography imaging was performed during arterial embolization, yielding an unexpected finding of bilateral ECAAs (Figure 1). The epistaxis resolved after embolization.

Given the patient's history of ectopia lentis and a positive family history, MFS was most likely the underlying etiology of her vascular abnormalities. Although she had no other physical exam findings indicative of MFS, her positive family history along with her ectopia lentis qualify for a diagnosis of MFS under the Revised Ghent criterion. ${ }^{9}$ The patient remains asymp- 


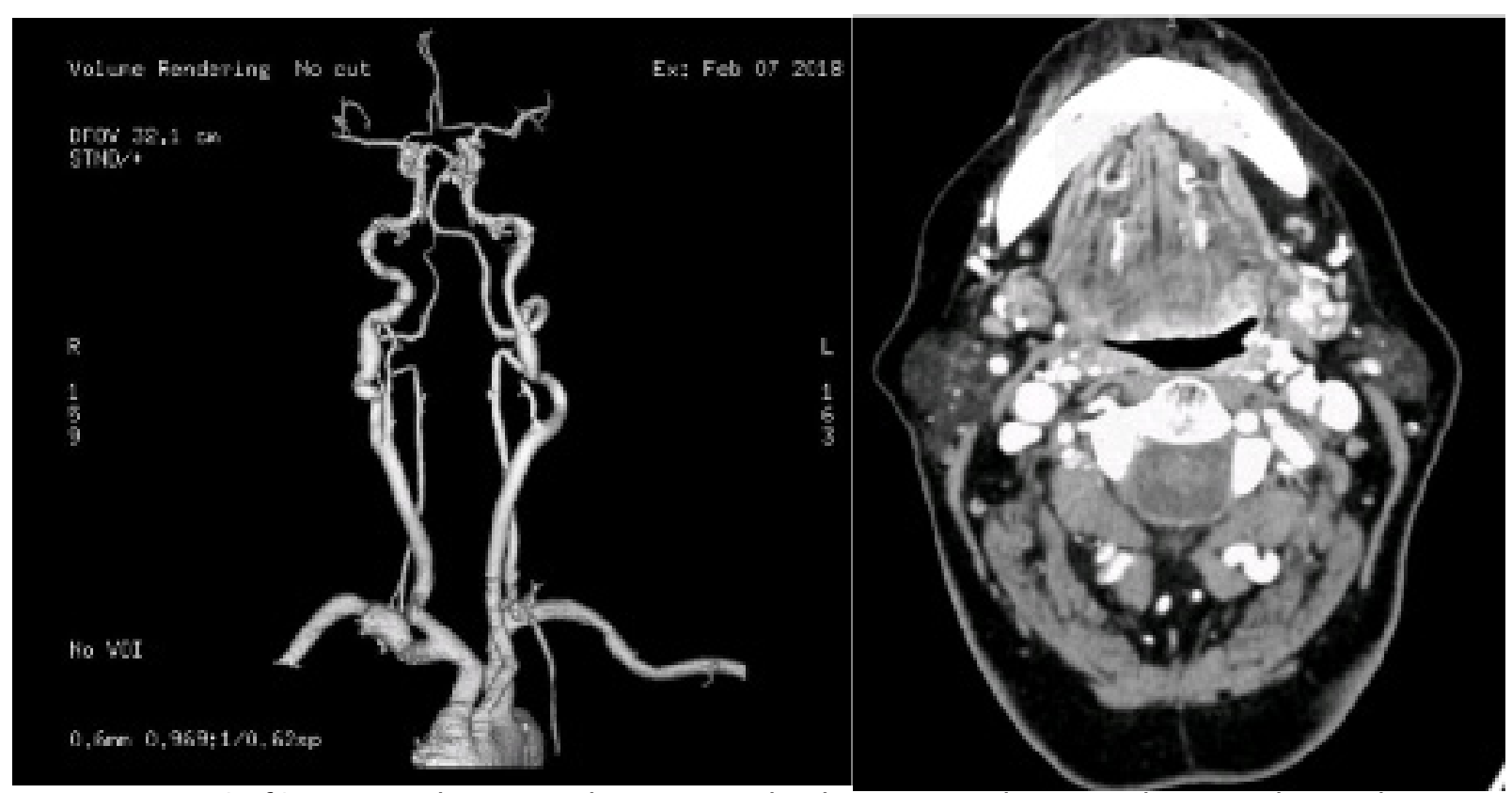

FIGURE 1. (Left) Computed tomography angiography shows 9.3- and 9-mm right internal carotid artery aneurysms in the mid and distal C1 segments, respectively, and a 9.7-mm left internal carotid artery aneurysm. (Right) This computed tomography angiography shows the $\mathrm{C} 1$ segment of the internal carotid artery at the aneurysm level with no evidence of dissection.

tomatic, and subsequent follow-up arteriograms at yearly intervals revealed no significant growth in the aneurysms over 2 years. There have been no additional aneurysms discovered in the patient.

\section{DISCUSSION}

ECAAs are exceedingly rare in patients with MFS, and to the best of our knowledge, only 10 total cases have been reported in the literature. ${ }^{8}$ In general, patients who develop ECAAs can present with a pulsatile mass, cerebral ischemic event, cranial nerve deficit, infection, dysphagia, tinnitus, bruit, hemorrhage, hoarseness, tracheal obstruction, dizziness, or without symptoms. However, in patients with MFS, the most common presentation is either asymptomatic or as a pulsatile cervical mass.,10 The pathogenesis by which MFS leads to aneurysmal dilation involves the loss in functionality of the protein fibrillin-1. This extracellular matrix glycoprotein is encoded by the FBN1 gene, which undergoes mutations in patients with MFS. Malfunction of fibrillin-1 causes a loss in elasticity of a patient's arterial wall, leading to a susceptibility to aneurysms. ${ }^{11}$ The histology of aneurysms in patients with connective tissue disease differ from those formed secondary to atherosclerotic disease, with the former consisting of cystic medial necrosis, loss of elastic fibers, and an increase in mucoid material. ${ }^{1,7}$

The treatment options for ECAAs include surgical or endovascular repair or conservative management, but there has yet to be a clear consensus on the best therapy. ${ }^{10,12}$ Surgical or endovascular interventions are typically recommended for symptomatic ECAAs, suspected infection, increasing aneurysm size, and absolute diameters greater than $2 \mathrm{~cm}$. If an ECAA is to be managed surgically, options include excision with primary anastomosis, excision with interposition graft, or extracranial-intracranial bypass. ${ }^{7}$ Historically, surgical treatment was by carotid ligation, but this is no longer common practice, as it is associated with a stroke risk of $25 \%$ and a mortality rate of $20 \% .5,12,14$ In a review by Kraemer and Zhou, complication rates from open surgery showed an early risk of mortality ranging from 0 to $7 \%$, perioperative stroke ranging from 0.7 to $11 \%$, and cranial nerve injury ranging from 0 to $66 \%$. Cranial nerve injuries included injuries to facial, vagus, spinal accessory, hypoglossal, and glossopharyngeal nerves. In spite of the risks, open surgery usually resulted in technical success. ${ }^{10,15}$ 
More recently, an endovascular approach has played an increasing role in the treatment of ECAAs. This approach consists of embolization of aneurysms with detachable coils and the use of stents or graft-covered stents. ${ }^{2}$ A systematic review on endovascular interventions found procedure success to be greater than $90 \%$ with incidences of stroke of $1.8 \%$, cranial nerve injury of $0.5 \%$, and overall in-hospital mortality of $4.1 \%$. The authors concluded that the high success rate and low complication rate for this approach make it feasible for continued exploration. ${ }^{16}$ However, patients with MFS may have anatomical differences, such as elongated internal carotid arteries, which may increase the risk for kinking or thrombosis after stent placement, and therefore, may have worse outcomes with this kind of intervention. ${ }^{8}$

Information regarding conservative therapy for ECAAs is best described by Fankhauser et al. ${ }^{6}$ Treatments include antiplatelet therapy, anticoagulation therapy, and serial imaging. Similar to our patient, most of the patients studied were asymptomatic but also had prior imaging that revealed the stability of the aneurysm. The authors demonstrated that treating patients medically resulted in no major morbidity or mortality related to the aneurysm.

\section{CONCLUSION}

ECAAs are an uncommon complication with varying presentations, etiologies, and management strategies. The recommendations in the literature for treatment of this pathology vary, and there seems to be no consensus at this time. While open surgery usually achieves clinical success, it is not without risk and may be unnecessary in asymptomatic patients. Endovascular approaches have been gaining popularity, but anatomical hurdles in MFS patients may hinder the use of this approach. Conservative follow-up of asymptomatic patients with serial imaging has the least risk; however, more research is necessary to determine the threshold for treatment as far as what constitutes dangerous growth of the aneurysm and what symptoms should prompt treatment with surgery or stents rather than antiplatelet agents. The uncommon presence of bilateral ECAAs only further complicates the choice of an aggressive management strategy, given that the risk of morbidity and mortal- ity with procedures on two arteries would likely be increased. Based on the current available evidence, patients should be apprised of the risks and benefits of treatment options and come to a joint decision with their healthcare provider.

\section{AUTHOR AFFILIATIONS}

1. Marshall University Joan C. Edwards School of Medicine, Huntington, West Virginia

\section{REFERENCES}

1. Latter DA, Ricci MA, Forbes RD, Graham AM. Internal carotid artery aneurysm and Marfan's syndrome. Can J Surg. 1989 Nov;32(6):463-6.

2. El-Sabrout R, Cooley DA. Extracranial carotid artery aneurysms: Texas Heart Institute experience. J Vasc Surg. 2000 Apr;31(4):702-12

3. Welling RE, Taha A, Goel T, Cranley J, Krause R, Hafner C, Tew J. Extracranial carotid artery aneurysms. Surgery. 1983 Feb;93:319-23.

4. McCollum CH, Wheeler WG, Noon GP, DeBakey ME. Aneurysms of the extracranial carotid artery: twenty-one years' experience. Am J Surg. 1979 Feb;137:196-200.

5. Veith CK, Tedesco JA, Landis GS. Bilateral extracranial internal carotid artery aneurysms: Case report and review of literature. Vascular. 2016 Oct;24(5):549-51.

6. Fankhauser GT, Stone WM, Fowl RJ, O'Donnell ME, Bower TC, Meyer FB, Money SR. Surgical and medical management of extracranial carotid artery aneurysms. J Vasc Surg. 2015 Feb;61(2), 389-93.

7. Sztajzel R, Hefft S, Girardet C. Marfan's syndrome and multiple extracranial aneurysms. Cerebrovasc Dis. 2001 11(4):346-49.

8. Sfyroeras GS, Nikolopoulou EA, Moulakakis KG, Lazaris AM, Kakisis JD, Geroulakos G. Extracranial internal carotid artery aneurysm in a patient with Marfan syndrome. Ann Vasc Surg. 2019 May;57:273.e7-273.e10.

9. Loeys BL, Dietz HC, Braverman AC, Callewaert BL, De Backer J, Devereux RB, Hilhorst-hofstee Y, Jondeau G, Fairve L, Pyeritz RE, Sponseller PD, Wordsworth P, De Paepe AM. The revised Ghent nosology for the Marfan syndrome. J Med Genet. 
2010 July;47(7):476-85.

10. Kraemer CJ, Zhou W. Carotid aneurysm review. Int J Angiol. 2019 Mar;28(1):17-19.

11. Cañadas V, Vilacosta I, Bruna I, Fuster V. Marfan syndrome. Part 1: pathophysiology and diagnosis. Nat Rev Cardiol. 2010 May;7(5),256-65.

12. Welleweerd JC, den Ruijter HM, Nelissen BGL, Bots ML, Kappelle LJ, Rinkel GJE, de Borst GJ. Management of Extracranial carotid artery aneurysm. Eur J Endovasc Surg. 2015 Aug;50(2):141-7.

13. Stanley J, Veith F, Wakefield T. Current therapy in vascular and endovascular surgery. 5th ed. Philadelphia:Saunders Elsevier. 2014 p.138-140.

14. Cooper A. Account of the first successful operation performed on the common carotid artery for aneurysm in the year of 1808 with postmortem examination in the year 1821. Guys Hosp Rep. 1836 1:53.

15. Zhou W, Lin PH, Bush RL, Peden E, Guerrero MA, Terramani T, Lubbe DF, Nguyen L, Lumsden AB. Carotid artery aneurysm: evolution of management over two decades. J Vasc Surg. 2006 Mar;43(3):493-96.

16. Li Z, Chang G, Yao C, Guo L, Liu Y, Wang M, Liu D, Wang $S$. Endovascular stenting of extracranial carotid artery aneurysm: a systematic review. Eur J Vasc Endovasc Surg. 2011 Oct;42(4):419-26. 